illumination, and the grinding process then has the advantage of removing the maker's name and other lettering which would otherwise be liable to appear in the field of view.

$$
\text { O. V. S. Heath }
$$

Research Institute of Plant Physiology,

Imperial College of Science and Technology, London, S.W.7. May 31.

${ }^{2}$ Barer, R., and Weinstein, W., J. Roy. Micr. Soc., 73, 148 (1953).

\section{Reaction of Stearic Acid Monolayers with Copper and Cuprous Oxide}

A NUMBER of workers have obtained results which indicate that only a portion of an adsorbed monolayer of stearic acid will react immediately with a copper substrate. (Of course, the reaction is actually with cuprous oxide since, at room temperature in air, all clean copper surfaces rapidly oxidize to cuprous oxide.) Bowden and Moor ${ }^{1}$ studied this reaction by putting stearic acid monolayers on radioactive copper and measuring the activity which could be removed by extraction with benzene. They found that when they placed a Langmuir-Blodgett monolayer of stearic acid on a copper surface and allowed it to stand for $18 \mathrm{hr}$. or heated it, only about $0 \cdot 14$ atom of copper reacts for every molecule of acid in the monolayer. They interpreted this as signifying that their procedure lost 75 per cent of the cupric stearate activity, due to adsorption on their equipment, since reaction was presumed to be complete. Consequently, they adopted an empirical correction factor of 4 .

Since the present work was begun, this interpretation was apparently refuted by Beischer's work ${ }^{2}$, in which he found that, if radioactive stearic acid is placed on a clean copper surface and the surface is heated until reaction occurs, initially only about 30 per cent of the stearic acid reacts. Here, there can be no possibility of the results being in error due to adsorption on apparatus since, in this technique, unreacted stearic acid is removed and the remaining stearic acid is measured. Therefore, Bowden and Moore are apparently mistaken in assuming that $\mathbf{a}_{0}$ Langmuir-Blodgett monolayer reacts completely. The molar ratio of copper to stearic acid $(0 \cdot 14-0 \cdot 30)$ seems to indicate quite clearly that, in the initial reaction of the Langmuir-Blodgett monolayer, about 30 per cent of the stearic acid reacts to give cupric stearate.

Bowden and Moore's results on the reaction of copper with a benzene solution of stearic acid were difficult to interpret quantitatively in this fashion. Therefore, experiments were carried out to measure the exact extent of the initial reaction of adsorbed monolayers. Clean copper foils were weighed on a microbalance, immersed in a 0.1 per cent solution of stearic acid in benzene, and the excess stearic acid washed off with benzene. After drying, the foils were reweighed, the increment representing the weight of stearic acid adsorbed. The foils were then extracted overnight with benzene in Soxhlet extractors and the extracts analysed for copper using a colorimetric method with sodium diethyl dithiocarbamate ${ }^{3}$. The foils were then weighed again. Since the final weights were equal to the initial weights minus the amount of cuprous oxide removed, the extraction must have removed virtually all the stearic acid and soap.

Analysis of the results indicated that the ratio of moles of copper reacted to moles of stearic acid adsorbed was approximately constant and equal to $0 \cdot 26 \pm 0.04$ for varying amounts of adsorbed acid. According to Preston and Bircumshaw ${ }^{4}$, when copper is oxidized in air at room temperature, the preferred orientation is for the (111) plane of the cuprous oxide to be parallel to the metal surface. In this plane, there is one copper atom for every $7 \cdot 8 \mathrm{A.}{ }^{2}$. Since the cross-sectional area of stearic acid in a monolayer is $21.0 \mathrm{A.} .^{2}$, each stearic acid molecule adsorbed on the cuprous oxide surface should have about $2 \cdot 6$ copper atoms available to it for chemical reaction. Even if one takes planes at somewhat different angles, the amount of copper per unit area will be at least enough to furnish a copper atom for each stearic acid molecule. It is therefore difficult to understand why only about one-quarter of the adsorbed stearic acid reacts, since thermodynamic calculations indicate that complete reaction should occur.

Of course, other planes will be exposed, and these may contain no copper atoms at all or may vary greatly in their rates of reaction with the monolayer. At present, however, no really satisfactory explanation of this phenomenon is available.

It is also of interest to see if copper itself will react with adsorbed stearic acid. Tingle ${ }^{5}$ has shown that when a copper surface is cut under a 1 per cent solution of lauric acid in paraffin oil, the fresh metal surface has a high coefficient of friction, which indicates that no reaction takes place. The previous experiments were repeated under an atmosphere of hydrogen, using copper foils which had been reduced in hydrogen at $450^{\circ} \mathrm{C}$. Blanks were run in the same way, merely omitting the copper foil. Application of the $t$ test to the results indicated that there was no significant difference between the blanks and the runs with copper. Thermodynamic calculations confirmed that metallic copper should not react with stearic acid in a non-oxidizing atmosphere.

We are indebted to Dr. E. A. Gulbransen for illuminating discussions on surface oxides. A full account of this work will be published elsewhere.

ATAN DOBRY

Henry E. Mahncke

Chemical Department,

Westinghouse Research Laboratories, East Pittsburgh, Pennsylvania. March 16.

${ }^{1}$ Bowden, F. P., and Moore, A. C., Trans. Farad. Soc., 47, 900 (1951). a Beischer, D. E., J. Phys. Chem., 57, 134 (1953).

Sandell, E. B. "Colorimetric Determination of Traces of Metals", 309 (Interscience Pub., Inc., New York, 2nd edit., 1950).

4 Preston, G. D., and Bircumshaw, L. L., Phil. Mag., vii, 20, 706 (1935).

${ }^{5}$ Tingle, E. D., Nature, 160, 710 (1947).

THE interesting results on partial reaction of the monolayer quoted by Dobry and Mahncke are of general interest and do in fact support the earlier observations of Bowden and Moore ${ }^{1}$. Even allowing for the factor of 4, they found that with copper surfaces immersed in a benzene solution of stearic acid the initial reaction was very slow, and after an immersion time of $5 \mathrm{~min}$. considerably less than a single monolayer of soap was formed (see their paper, footnote p. 904). After prolonged immersion, chemical attack was quite heavy and corresponded to the reaction of several molecular layers. Results of this type obtained with layers adsorbed from solution are probably more significant than those obtained with layers deposited from the Langmuir trough. The latter are often unstable and tend to collapse; they appear to form bridges over the surface irregularities so that the area of molecular contact may be 